\title{
Sleep-apnea and cardiomyopathy in acromegalic patients
}

Authors: Ruth Sánchez-Ortiga ${ }^{1}$, Alfredo Candela ${ }^{2}$, Vicente E. Climent ${ }^{3}$, Laura Sánchez-Tejada ${ }^{4}$, Irene Monjas-Cánovas ${ }^{5}$, Javier Abarca ${ }^{6}$, Oscar Moreno-Perez ${ }^{1}$, Antonio Picó ${ }^{1}$.

Institutions: 1. Endocrinology Department, 2. Respiratory Department. 3. Cardiology Department, 4. Research Unit, 5. Head and Neck Department, 6. Neurosurgery Department; Hospital General Universitario de Alicante

Introduction: Sleep disordered breathing (SDB) promotes structural myocardial changes and can trigger cardiac arrhythmias. Acromegalic patients have high prevalence of SDB and GH-IGF-I excess is related to a specific cardiomyopathy characterized by concentric cardiac hypertrophy and diastolic dysfunction.

The aim of this study was to assess the relacionship between SDB and cardiac dysfunction on acromegalic patients.

Materials and methods: Observational descriptive study of 32 acromegalic patients and 20 patients referred to the respiratory department for SDB study paired with acromegalic patients in sex, age and BMI. Polysomnography, echocardiography and electrocardiography (ECG) were performed in all patients. Patients were defined having sleep-apnea (SA) if they had more than ten apneas or hypopnoeas per hour. Statistical analysis: Qualitative data are expressed as percentage and absolute number; quantitative data are expressed as medium \pm SD. Pearson, T-Student and X2 tests were used; statistical significance $p<0.05$

Results: There were no differences between acromegalic and controls on baseline characteristics (exposed on table 1), except for respiratory abnormalities that were more prevalent on controls ( $3 \% \mathrm{vs.} 35 \%, \mathrm{p}=0.02)$. Twenty-three acromegalic patients were under somatostatin analogs therapy (SA), differences with patients without treatment are exposed on table 2 .

Table 1. Baseline characteristics

\begin{tabular}{ccc}
\hline & $\begin{array}{c}\text { Acromegalic } \\
(\mathrm{n} \mathrm{32})\end{array}$ & $\begin{array}{c}\text { Controls } \\
(\mathrm{n} \mathrm{20})\end{array}$ \\
Men (n) & $43.8 \%(14)$ & $50 \%(10)$ \\
Age (years) & $50.3 \pm 11.4$ & $53.2 \pm 12.7$ \\
BMI (kg/m2) & $29.4 \pm 4.8$ & $31.6 \pm 6.6$ \\
Hypertension (n) & $46.9 \%(15)$ & $50 \%(10)$ \\
Hyperchol. $(\mathrm{n})$ & $34.4 \%(11)$ & $45 \%(9)$ \\
DM $(\mathrm{n})$ & $9.4 \%(3)$ & $15 \%(3)$ \\
Thyroid disease $(\mathrm{n})$ & $39.6 \%(13)$ & $15 \%(3)$ \\
CVD $(\mathrm{n})$ & $34.4 \%(11)$ & $15 \%(3)$ \\
Smoker $(\mathrm{n})$ & $50 \%(16)$ & $70 \%(14)$ \\
\hline
\end{tabular}

Table 2. Acromegalic patients

\begin{tabular}{cccc} 
& SA & Non-SA & \\
$(\mathrm{n} \mathrm{23})$ & $12.5 \%(1)$ & 0.09 \\
Men (n) & $52.2 \%(12)$ & $61.4 \pm 11.2$ & $\mathbf{0 . 0 0 1}$ \\
Age $(\mathrm{y})$ & $46.6 \pm 8.9$ & $27.7 \pm 2.9$ & 0.21 \\
$\mathrm{BMI}(\mathrm{kg} / \mathrm{m} 2)$ & $30.2 \pm 5.2$ & $0.9(0.8-1.9)$ & 0.06 \\
$\mathrm{GH}(\mathrm{ng} / \mathrm{ml})$ & $4.9(1.65-12.3)$ & 0.006 \\
$\mathrm{IGF}-1(\mathrm{ng} / \mathrm{ml})$ & $364(253-854)$ & $160(114-281)$ & $\mathbf{0 . 0 0 6}$ \\
Evolution $(\mathrm{m})$ & $59.8 \pm 46$ & $104.6 \pm 72.9$ & 0.07 \\
Surgery & $69.6 \%(16)$ & $100 \%(8)$ & 0.15 \\
Radiotherapy & $56.5 \%(13)$ & $12.5 \%(1)$ & $\mathbf{0 . 0 4 5}$ \\
DM (n) & $13.0 \%(3)$ & 0 & 0.59 \\
\hline
\end{tabular}

DM: diabetes mellitus; CVD: cardiovascular disease.

$24(75 \%)$ acromegalic patients and $15(78.9 \%)$ controls had SA, all of them due to obstructive cause. 18 (58.1\%) acromegalic patients and $6(30 \%)$ controls had diastolic dysfunction of left ventricle $(\mathrm{p}=0.05)$. Patients with/without SSA didn't have different prevalence of SA or cardiac dysfunction.

Only acromegalic patients, but not controls, with SBD compared with those without SBD had higher diastolic LV diameter (Fig. 1) and higher pulmonary artery systolic pressure (Fig. 2).

Moreover, they had a trend toward less cardiac frequency (72.6 \pm 7.7 vs $78.1 \pm 9.1, p=0.08)$, less ejection fraction $(62.6 \pm 12.7$ vs $71.4 \pm 10.4, \mathrm{p}=0.09)$ and more alterations on ECG (50\% vs $12.5 \%$, p 0.09).

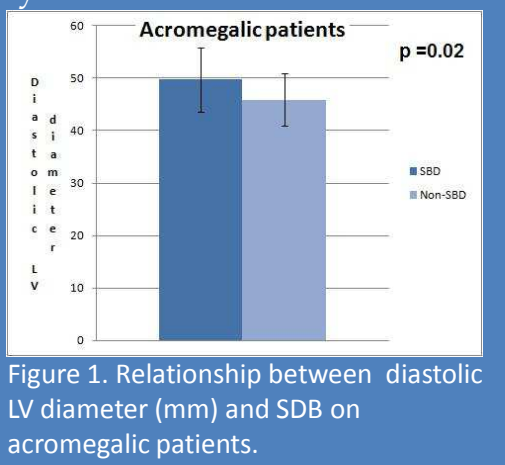

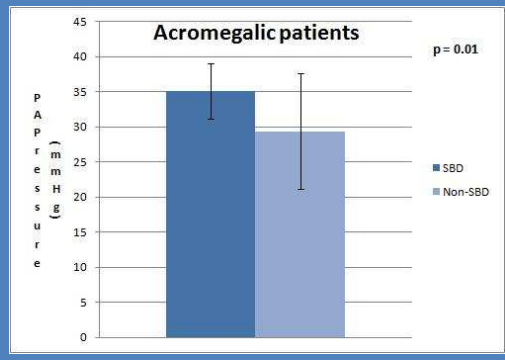

Figure 2. Relationship between pulmonary artery systolic pressure and SBD on acromegalic patients.

Conclusion: SDB is a risk factor to cardiac abnormalities in acromegalic and non acromegalic people. The prevalence of cardiac abnormalities in acromegaly is higher when SA is present, independent of the cure or control of acromegaly. 